\title{
Defined Bilayer Interactions of DNA Nanopores Revealed with a
}

\section{Nuclease-Based Nanoprobe Strategy}

\author{
Jonathan R. Burns*, Stefan Howorka* \\ Department of Chemistry, Institute of Structural Molecular Biology, University College London, \\ London WC1H OAJ, United Kingdom
}

KEYWORDS: DNA, nanopore, membrane topography, exonuclease, membrane biophysics 


\section{ABSTRACT}

DNA nanopores are a recent class of bilayer-puncturing nanodevices that can help advance biosensing, synthetic biology, and nanofluidics. Here, we create archetypal lipid-anchored DNA nanopores and characterize them with a nanoprobe-based approach to gain essential information about their interactions with bilayers. The strategy determines the molecular accessibility of DNA pores with a nuclease and can thus distinguish between the nanopores' membrane-adhering and membrane-spanning states. The analysis reveals, for example, that pores interact with bilayers in two steps whereby fast initial membrane tethering is followed by slower reorientation to the puncturing state. Tethering occurs for pores with one anchor, whilst puncturing requires multiple anchors. Both low and high-curvature membranes are good substrates for tethering, but efficient insertion proceeds only for high-curvature bilayers of the examined lipid composition. This is likely due to the localized lipid misalignments and the associated lower energetic barrier for pore permeation. Our study advances the fields of DNA nanotechnology and nanopores by overcoming the considerable experimental hurdle of efficient membrane insertion, by providing mechanistic insights to aid the design of advanced nanopores, and by offering a useful route to probe bilayer orientation of DNA nanostructures. 
Nanopores puncture lipid bilayers via nanoscale holes to facilitate defined transport of molecular cargo. Nanopores are essential in biology and play an important role in shuttling biomolecules between membrane compartments and cells. ${ }^{1-7}$ Outside biology, nanopores have achieved a break-through in portable DNA sequencing ${ }^{8,9}$ and additionally found widespread use as tools to detect and characterize individual molecules or nanoparticles. ${ }^{10-20}$ The underlying sensing principle relies on the passage of individual objects through an electrolyte-filled pore that leads to transient detectable changes in electrical read-out. To function efficiently, a pore should allow passage of one molecule at a time. In order to help expand the nanopore sensing field, customizable pores require tunable pore dimensions and pore chemistries, along with detailed structural and chemical characterization.

Nanopores assembled from folded DNA are the most recent class of the membrane-spanning nanodevices. ${ }^{21-24}$ Their key advantage is the ease with which they can be rationally designed and generated. $^{25-31}$ Unlike proteins or peptides, DNA is a building block with highly predictable folding properties. ${ }^{32-36}$ Consequently, the construction of DNA pores is simpler and can harness the power of DNA nanotechnology. ${ }^{25}$ For example, dedicated design software automatically provides the sequence of component oligonucleotides for a user-defined target DNA nanostructure with chosen geometry and dimensions. ${ }^{37}$ It is also possible to engineer tunable nanoscale movement, something which would be much harder to do in protein engineering. ${ }^{38}$ To achieve membrane anchorage, the negatively charged DNA nanostructures are modified with hydrophobic tags, as demonstrated by single-channel current recording or fluorophore transport measurements. ${ }^{22,23,26,30}$ DNA nanopores do not, however, efficiently insert into bilayers typically used for electrical recordings. While the low insertion frequency is understandable in light of the DNA nanostructures' highly negative charged nature, it is nevertheless a problem in experiments 
that can lead to lower through-put and statistically less supported data sets. Furthermore, little is known about the insertion mechanism of DNA pores, while several protein pores can insert via a two-step mechanism involving a membrane-bound pre-pore and lies with an extramembrane capregion on the bilayer which then undergoes a conformational change so that proteins loops puncture the lipid bilayer. $^{39}$

Understanding the interaction of DNA nanopores with lipid bilayers is of scientific and technological interest. In practical terms, a greater understanding can help increase the efficiency of DNA pore insertion and the through-put of investigations. At a more fundamental level, an indepth study can answer several key questions about nanopore-bilayer interactions. For example, what is the mechanism by which DNA nanopores insert into bilayers? And do pores quantitatively insert into membranes? Furthermore, what is the influence of the number of lipid anchors for achieving membrane anchoring? And what role does membrane curvature play for insertion? This latter question is motivated by the known role of membrane curvature in the binding and activity of membrane proteins in biology ${ }^{40,41}$ including those with BAR domains. ${ }^{42}$ Previous studies on DNA nanopores have primarily focused on their structure and dynamics, yet the interaction with bilayer has, so far, primarily explored with powerful computational methods $\mathrm{s}^{31,43}$ which are nevertheless limited by their time constraints and exclude mechanistic questions.

In this report, we answer the key questions about bilayer interaction for an archetypical DNA nanopore. The pore is composed of six hexagonally arranged duplexes that are assembled from six single stranded DNA oligonucleotides. ${ }^{38}$ Based on the design, the duplexes are interconnected at their termini with hairpin junctions (Figure 1A, SI Tables S1 and S2, and Figure S1 and S2). The barrel-shaped pore measures $9 \times 5 \times 5 \mathrm{~nm}$ in height, depth and width, 
respectively, with an inner channel diameter of around $2 \mathrm{~nm}$. Up to three of the DNA duplexes carry cholesterol membrane anchors for bilayer insertion. The anchors are positioned around the central perimeter of the DNA barrel and are covalently attached at the 3 ' end of the DNA strands (Figure 1A and Figure S1). The pore with no, one, two, and three cholesterol tags are termed NP0C, NP-1C, NP-2C, and NP-3C (Figure 1A). While NP-3C can span the membrane, ${ }^{38}$ the anchoring of NP-2C and NP-1C is unknown; NP-0C serves as a negative control (Figure 1A).
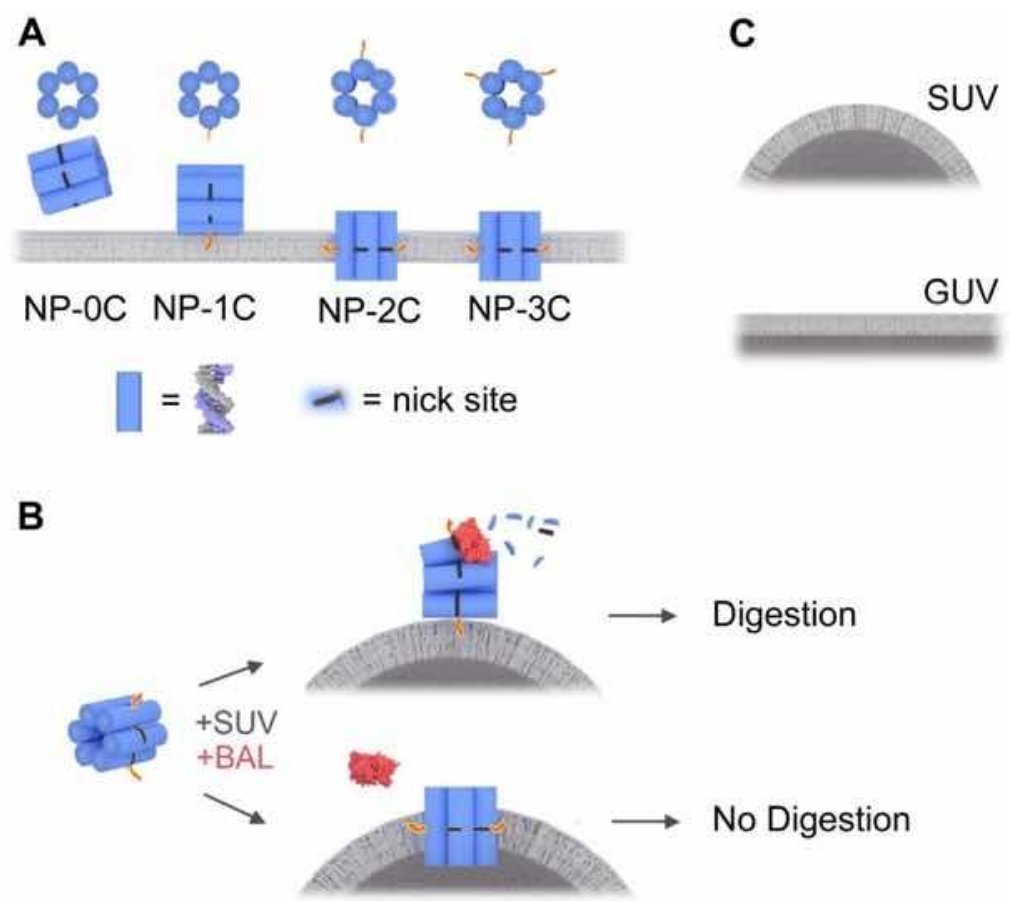

Figure 1. An archetypal cholesterol-tagged DNA nanopore used in this study, and the nuclease probe assay employed to determine the orientation of DNA nanopores relative to bilayer membranes. (A) Top and side view of barrel-shaped DNA pores composed of six interconnected duplexes (blue) with either 0, 1, 2 or 3 cholesterol membrane-anchoring tags (orange), and their proposed interaction with linear bilayers (grey) by adhering to the membrane (NP-1C) or spanning it (NP-3C). The centrally located black lines indicate nicks in the DNA duplexes. The pores' six DNA strands are each 50 bases long thereby forming a 21 base pair-long duplexes 
with 4 thymidines in the interduplex hairpins to prevent blunt-end stacking interactions between two or more pores. ${ }^{38}$ (B) In the nuclease probe assay, free and membrane-adhering nanopores with exposed DNA nicks are digested and fragmented by BAL-31 (red), while membranespanning pores with DNA nicks positioned in the membrane are sterically shielded from digestion. (C) Lipid vesicles used within the probe assay are giant unilamellar vesicles (GUVS) with a low curvature of $0.2 \mu m^{-1}$ and small unilamellar vesicles (SUVs) with a high curvature of $20 \mu m^{-1}$.

To answer the questions regarding DNA nanopore-bilayer interactions, we developed a probebased approach. Our strategy determines the orientation and topography of DNA nanopores at bilayers with a nuclease probe in combination with fluorescence read-out (Figure 1B). The probe can distinguish between nanopores in a membrane-adhering and hence nuclease-accessible orientation, as opposed to nanopores that are bilayer-inserted and sterically protected from DNA digestion (Figure 1A and 1B). The nuclease probe used in the assay is exonuclease BAL-31 that digests preferentially dsDNA at nicks in the phosphodiester backbone (Figure 1A and 1B, black lines). ${ }^{4-45}$ Since the nicks of the nanobarrels' duplex are located close to the lipid anchors, and therefore situated in the membrane-spanning region, BAL-31 is expected to distinguish between a membrane-spanning state, where the nicks are shielded by the surrounding lipid bilayer and protected from nuclease digestion, and a membrane-adhering tethered state, where the nicks are more readily exposed to the enzyme. To probe the influence of membrane curvature on nanopore insertion, the nuclease probe assay was carried out with small unilamellar vesicles (SUVs) composed of diphytanoyl-sn-glycero-3-phosphocholine with a high positive curvature of $20 \mu \mathrm{m}^{-1}$ and giant unilamellar vesicles (GUVs) with a 100-fold lower curvature with the same lipid composition (Figure 1C). 


\section{RESULTS/DISCUSSION}

DNA nanopores with up to three cholesterol anchors are formed efficiently. The nanostructures were generated by annealing the six component single stranded oligonucleotides (sequences in Table S1 and S2, Figure S1). ${ }^{38}$ Analysis of the assembly mixture by gel electrophoresis showed the nanostructures were successfully folded (Figure S3) since nanopores without and with one cholesterol anchor (NP-0C, NP-1C, respectively) migrated as defined single bands (Figure S3). Due to strong cholesterol-gel matrix interactions, NP-3C with 3 hydrophobic lipid anchors did not migrate out of the loading pocket and into the gel (Figure S3). ${ }^{22}$ Addition of a mild detergent is known to suppress the gel matrix interactions. ${ }^{38}$ Additional gel analysis established that DNA nanopores are stable towards DNAase I enzyme at physiological concentrations (Figure S4). This is important for potential applications of DNA nanopores in cell biology and biomedicine including bodily fluids.

\section{The nuclease probe assay translates digestion of DNA nanopores into a clear fluorescence}

signal. We determined the conditions under which the nuclease probe digest DNA nanopores efficiently. The experiments were first carried out in the absence of membranes. BAL-31 exonuclease (9.6 U) was added to solubilized NP-0C, NP-1C and NP-3C, and the digestion extent was monitored using UV absorption spectroscopy. Addition of the enzyme caused an increase of the DNA's absorbance band at $260 \mathrm{~nm}$, indicating fragmentation of the six-duplex pore (Figure 2A, Figure S5). The digestion profiles for NP-0C, NP-1C and NP-3C were similar, implying that the enzyme's activity was not significantly altered by the presence of the cholesterol lipid anchors (Figure 2A, Figure S5). Structural digestion of the nanobarrels was confirmed by UV melting profile analysis, with and without the addition BAL-31 (Figure S5). 
Fast digestion of the DNA pore NP-0C was also shown by agarose gel electrophoresis and subsequent quantification of the gel band intensities (Figure 2B). However, electrophoresis was not suitable to monitor lipid-anchored pores given their interaction with the gel matrix, while UV spectroscopy had a low sensitivity in the $\mu \mathrm{M}$ range.
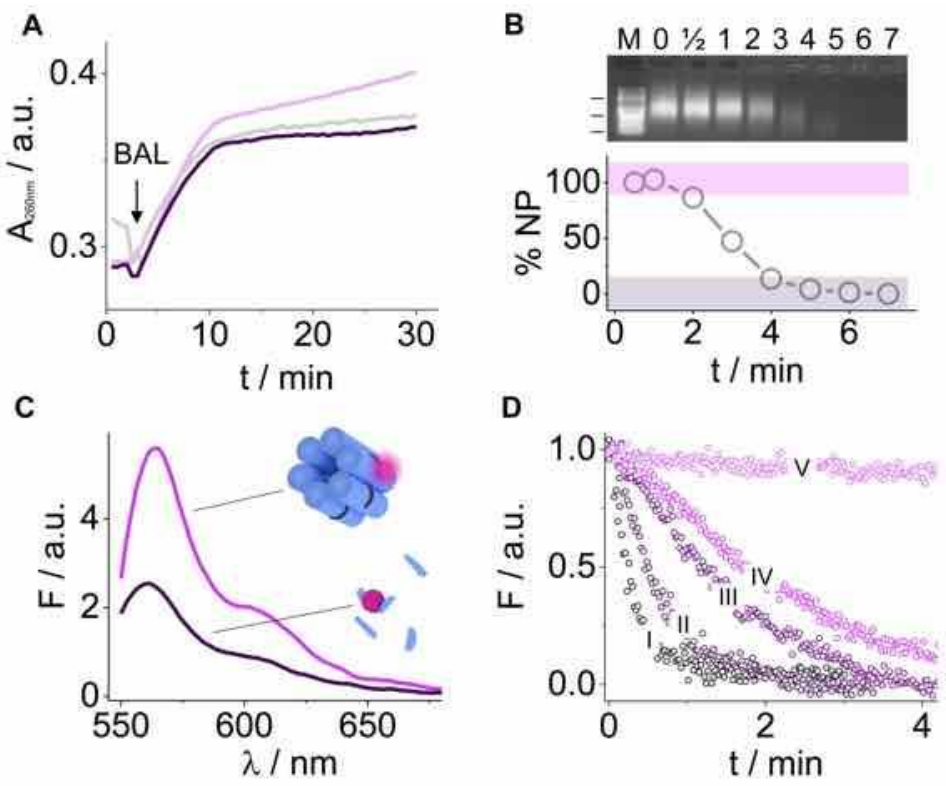

Figure 2. The nuclease probe assay leads to the digestion of DNA pores and a corresponding clear fluorescence signal. (A) Kinetic profiles for the nuclease probe-mediated digestion of NPOC (purple), NP-1C (grey) and NP-3C (pink) as monitored by absorbance at $260 \mathrm{~nm}$. The arrow indicates addition of $9.6 U$ of BAL-31. (B) Agarose gel electrophoretic analysis of NP-0C incubated with 9.6 U of BAL-31 for various durations (top). 100 bp marker shown on the left. The indicated positions represent 1000, 500 and $100 \mathrm{bp}$. The intensities of gel bands are shown below the gel. The light purple and dark purple indicate the intact, and digested pores, respectively. (C) Fluorescence emission spectra of Cy3-labelled NP-OC before (light purple) and after (dark purple) addition of BAL-31 (1.7 U). (D) Kinetic digestion profiles of Cy3-labelled 
NP-OC with decreasing amounts of BAL-31 (I-V, 13, 6.6, 1.7, 0.7 and 0.1 U of BAL-31, respectively).

To attain a universal and sensitive read-out for digestion, a fluorescent reporter was incorporated into the DNA nanostructures. Cy3 was selected due to its environment-sensitive properties which lead to significant fluorescence changes depending on the dye's attachment to a duplex, single strand, or isolated nucleotides, or in an unbound state. The fluorophore was linked to the 5' phosphate of a component DNA strand. Addition of BAL-31 (1.7 U) to Cy3-labeled NP0C resulted in a 54\% decrease in emission intensity within $5 \mathrm{~min}$ (Figure 2C). This reduction was most likely due to quenching interactions that arise when $\mathrm{Cy} 3$ is no longer conformationally locked by the duplex but -after digestion- is free to bind to the surrounding nucleobases, or selfquench by photodisomerisation. Monitoring $\mathrm{Cy} 3$-fluorescence emission as a function of digestion time gave rise to the expected exponential decay profiles (Figure 2D). The profiles were also dependent on the concentration of BAL-31 (0.1-13 U) but plateaued after 5 min, indicating complete digestion of the DNA nanostructure (Figure 2D). A minimal concentration of enzyme $(0.1 \mathrm{U})$ resulted in a much slower decrease in emission; however, complete digestion was achieved at approx. $120 \mathrm{~min}$ (Figure S6).

DNA nanopores bind with at least one cholesterol anchor to the bilayer membrane. The binding of lipid-modified DNA nanopores was analyzed with gel electrophoresis. Only one cholesterol anchor was required for membrane anchoring, as demonstrated by the upshift of the gel-band of NP-1C upon SUV incubation. The bilayer-bound DNA pores were upshifted as vesicles were too large to migrate into the gel matrix (Figure 3A). Increasing amounts of SUVs resulted in a greater fraction of nanopore binding (Figure 3A and 3B). Complete vesicle binding was achieved at a molar ratio of $1: 10$ for NP to SUV, and this ratio was subsequently used for 
the nuclease probe studies. Complete vesicle binding also confirmed the high homogeneity of the DNA nanopore preparation and ruled out the possibility that a small fraction of the DNA pores had their anchor in an inaccessible, non-membrane binding conformation. In a control experiment, nanopores without lipid anchors did not interact with SUVs, and no gel shift occurred (Figure 3A and 3B). Due to strong cholesterol-gel matrix interactions, NP-3C with 3 hydrophobic lipid anchors did not migrate out of the loading pocket and into the gel (Figure S7), therefore no binding information could be extracted reliably from this construct. ${ }^{22}$
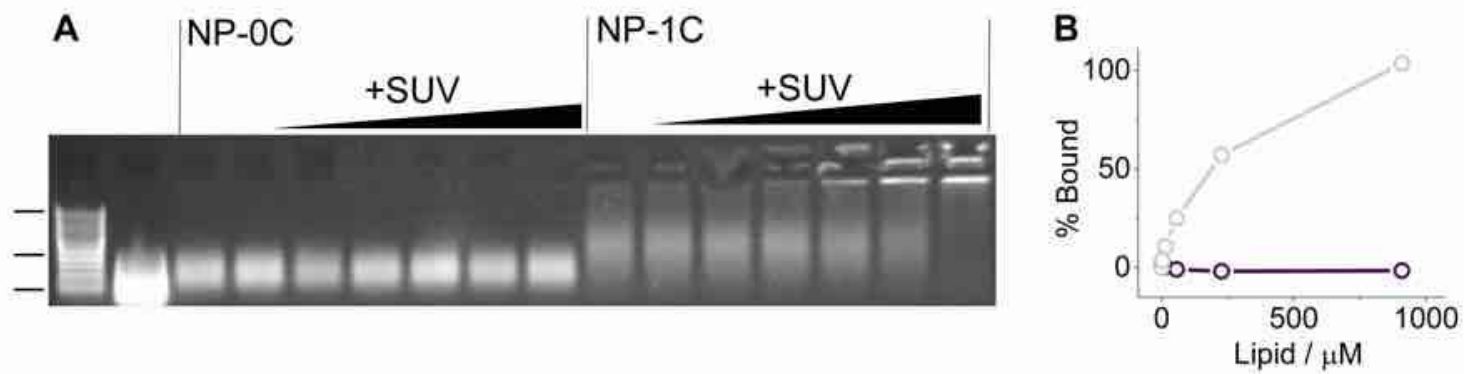

Figure 3. Concentration-dependent binding of DNA nanopores to SUV membranes. (A) 0.5\% Agarose gel electrophoretic analysis of NP-1C binding to SUV added at increasing concentrations (0, 0.9, 3.6, 14.0, 56.8, 227 and $909 \mu M$ lipid from left to right). NP-OC does not bind as it lacks the cholesterol anchor. $1 \mathrm{kbp}$ and $100 \mathrm{bp}$ markers shown on the left. The lines indicate the bands for the 10, 3 and $0.5 \mathrm{kbp}$ markers. (B) Plot summarizing the extent of binding of NP-OC (purple) and NP-1C (grey) to SUVS as analyzed with gel electrophoresis in panel A. The ratio of NP to vesicles was calculated from the known stock concentrations for NP and SUVs-lipids of $1 \mu M$ and $1 \mathrm{mM}$, respectively. Using the given volumes yields a final lipid concentration of $909 \mu M$ under the known composition of around 100,000 lipids per SUV of 100 nm diameter. This translates into a maximum binding ratio of 10 NPs per SUV. 
The nuclease probe assay reveals the orientation of lipid-anchored DNA nanopores relative to the membrane. To establish whether DNA nanopores bind to membranes in adhering or spanning orientations, NP-0C, NP-1C, NP-2C and NP-3C were mixed with SUVs and subjected to the nuclease probe assay. In the absence of the lipid vesicles, the membrane anchor did not influence nuclease digestion as implied by the similar decreases in Cy3 emission (Figure 4A). However, pre-incubation of DNA pores with lipid vesicles for $60 \mathrm{~min}$ strongly altered the digestion profiles (Figure 4B). Membrane-bound NP-1C and NP-3C underwent slower, or almost no digestion, respectively, when compared to the fast fragmentation of non-membrane bound NP-0C. Quantitative analysis of the digestion profile by single exponential fitting yielded halflife, $\tau$, values and $5.6 \pm 3.0 \mathrm{~min}$ for NP-1C, $38.5 \pm 3.8 \mathrm{~min}$ and $122 \pm 29 \mathrm{~min}$ for NP-2C and NP$3 \mathrm{C}$, respectively, and $2.5 \pm 0.3 \mathrm{~min}$ for NP-0C $(n=3)$. The differences in $\tau$ for NP-1C and NP3C are interpreted to reflect two membrane orientations. Pores with one cholesterol tag resulted in intermediate nuclease protection, and are inferred to tether to membranes in a parallel orientation where the neighboring vesicle bilayer only partly shields the DNA pore from the nuclease probe. However, NP-3C with three cholesterols tags drastically increased in nuclease protection, and therefore likely spans the bilayer membrane as the nuclease-targeting DNA nicks within the pore are protected by the surrounding lipid bilayer. The membrane-spanning state of NP-3C was confirmed by single-channel electrical recordings where the flow of ions through the pore is recorded as a function of time (Figure 4C,D). ${ }^{38}$ As expected, addition of BAL-31 did not interfere with the current recordings as the sites for nuclease attack -the DNA nicks- were embedded within the lipid bilayer (Figure 4C,D, Figure S8). However, an about 10-fold higher BAL-31 concentration led to the digestion of the membrane-inserted NP-3C pore (Figure S9) likely as BAL-31 also has a less prominent endonuclease activity. ${ }^{44,45}$ 

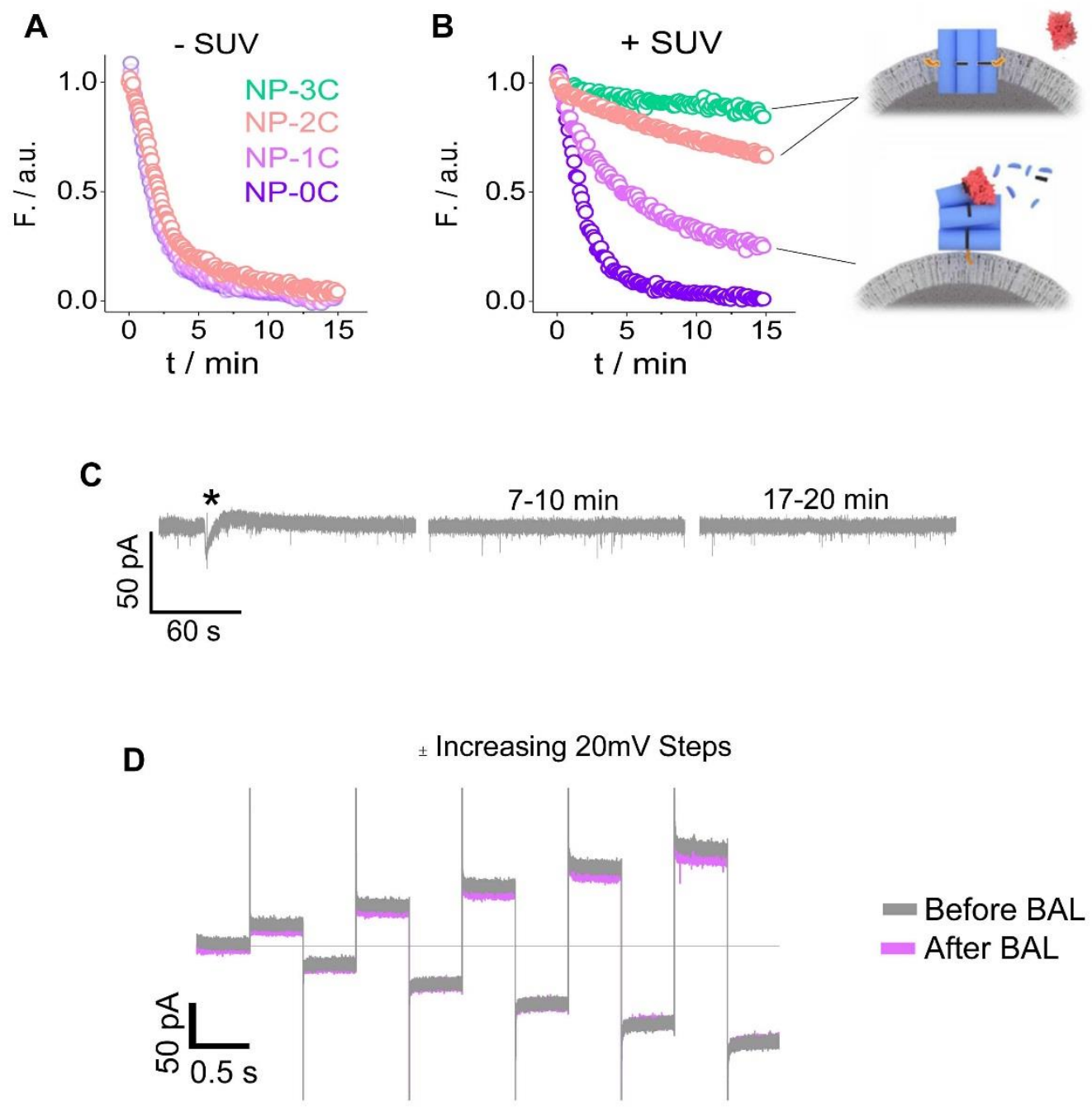

Figure 4. Membrane-adhering and membrane-spanning DNA nanopores are distinguished with the nuclease probe assay. $(\boldsymbol{A}, \boldsymbol{B})$ Kinetic fluorescence plots of Cy3-labelled DNA nanpores, NPOC (red), NP-1C (blue), NP-2C (red) and NP-3C (green), after addition of BAL-31 (1.7 U) and incubation for 60 min in the absence $(\boldsymbol{A})$ and presence $(\boldsymbol{B})$ of SUVs. (C) Single channel current recording of NP-3C nanopores, before and after the addition of BAL-31 (1.7 U). The astrix indicates the addition of the enzyme. (D) Current traces with stepped voltages for single NP-3C pores, before and after the addition of BAL-31. 
The membrane-spanning orientation of NP-3C is established with the nuclease probe assay and a pore variant. To confirm that the increased nuclease protection for NP-3C indeed reflects its membrane spanning orientation, a pore variant $\left(\mathrm{NP}-3 \mathrm{C}^{4}\right)$ with a differently positioned nuclease attack site at duplex 4 was employed. While the attack site of the normal pore is in the vertical middle of the duplex, the DNA nick in pore variant $\mathrm{NP}-3 \mathrm{C}^{4}$ is designed to be $3 \mathrm{~nm}$ off this central hydrophobic anchor region (Figure 5A, Figure S1, Tables S1 and S2). Consequently, moving the nick site outside the likely membrane-inserted region should increase the nick's molecular accessibility to the nuclease probe and hence increase fragmentation.

A

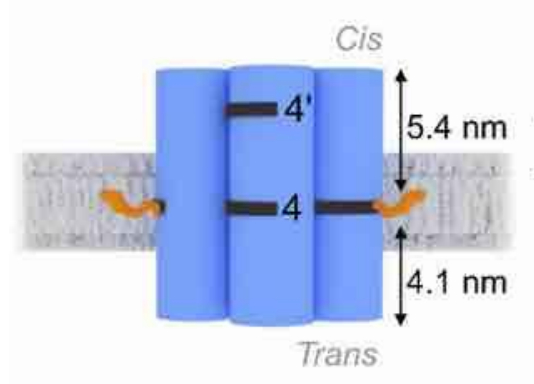

B

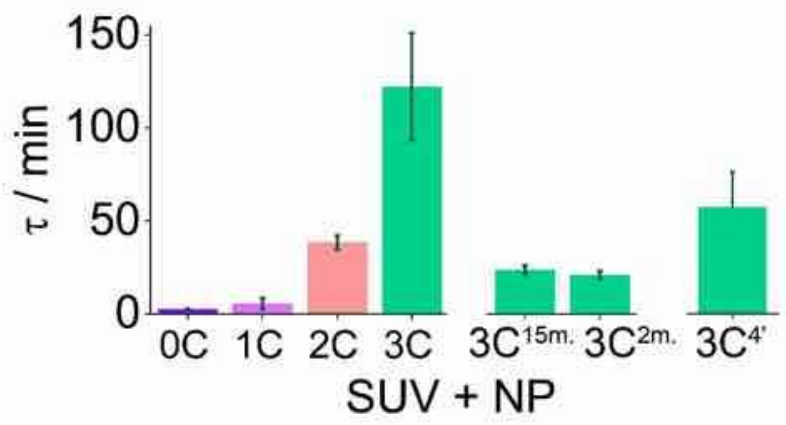

Figure 5. Confirming the membrane-spanning orientation of DNA nanopores with an NP-3C variant. (A) Schematic drawing of bilayer-bound NP-3C pore containing the duplex nick either at the lipid anchors (4), or positioned towards the duplex terminus (4'). The subtle asymmetry of the lipid anchor positioning along the long DNA axis is annotated cis and trans. (B) Kinetic $\tau$ values for the digestion of $N P-0 C, N P-1 C, N P-2 C$ and $N P-3 C$ in the presence of SUVs from Fig. $4 B$, and $N P-3 C^{4}$ incubated for $60 \mathrm{~min}$ in the presence of SUVS. For comparison, the values for NP-3C incubated with SUVs for 15 and 2 min are also shown. 
Fluorescence read-out of the digestion assay revealed that moving the nuclease attack site out of the membrane led to a strong decrease of nuclease digestion (Figure 5B, Figure S10). Using the measure of half-life $\tau$, the pore variant $\mathrm{NP}-3 \mathrm{C}^{4}$ had a value of $18.5 \pm 4.8$ min which is 6.6 times faster than for NP-3C. This result strongly suggests that, first, the nick in pore variant $\mathrm{NP}-3 \mathrm{C}^{4}$ is positioned outside the lipid bilayer region, and, second, the nanopore with three cholesterol anchors is inserted in a membrane-spanning orientation.

Nanopore NP-3C inserts via a two-step mechanism into highly curved membranes. After establishing that the slow digestion reflects the membrane-spanning state, we investigated the mechanism of pore insertion. In principle, the DNA nanopores NP-3C with three anchors can either insert directly in a single step into the membrane. Alternatively, the pore can insert via a two-step mechanism, first bind in a membrane-adhering orientation to the membrane, and subsequently re-orient itself to insert into the bilayer to span it. We discriminated between the single and two-step model by varying the duration of the pre-incubation of nanopores and SUVs prior to subjecting the mixture to the nuclease probe assay. The reasoning was that a shorter duration would lead -in case of two-step mechanism- to a high proportion of membraneadhering pore that had not yet re-oriented and inserted into the bilayer. Indeed, pre-incubation times of 2 and 15 min showed relatively quick digestion (Figure S11) when compared to the slow fragmentation after $60 \mathrm{~min}$ pre-incubation (Figure 4B, Figure 5). The faster digestion after short incubation implies a membrane-adhering orientation, and hence strongly supports that DNA nanopores follow a two-step mechanism of first fast membrane binding followed by slower insertion into the bilayer. The two-step process with final insertion is also supported by data on $\mathrm{NP}-3 \mathrm{C}^{4}$. 
NP-3C inserts poorly into low-curvature membranes. To study the effect of membrane curvature on the nanopores insertion, we complemented the established results with highcurvature SUVs with data on low-curvature GUVs. Membrane-orientation of DNA nanopores was determined with the nuclease probe assay as above, but the fluorescence-read out of digestion relied on directly visualizing GUVs with confocal laser scanning microscopy. Microscopy confirmed GUV-binding for NP-1C and NP-3C (Figure 6A). The two pore types had been differently labelled with Cy3 and FAM (Figure S12) and hence allowed simultaneous monitoring on the same GUV. Both NP-1C and NP-3C gave rise to well-defined membrane halos which strongly suggested effective binding on GUVs. The addition of the nuclease probe (1.7 U BAL-31) resulted in rapid and complete digestion of both NP-1C and NP-3C within 5 min (Figure 6A). Plotting the fluorescence intensity of the membrane halos over time revealed comparable digestion rates for both pore types (Figure 6B). The high susceptibility of NP-3C to digestion implies that this pore was predominately orientated in a membrane tethered state of GUV membrane (Figure 6C). The GUV and SUV results highlight the effect of membrane curvature on the nanopores' insertion. To confirm that nanopores are indeed not spanning the bilayer membrane, NP-3C was encapsulated inside GUVs and proven not to be digested when the nuclease probe was added to the exterior, even at a considerable excess (Figure 6A and S13). 
A

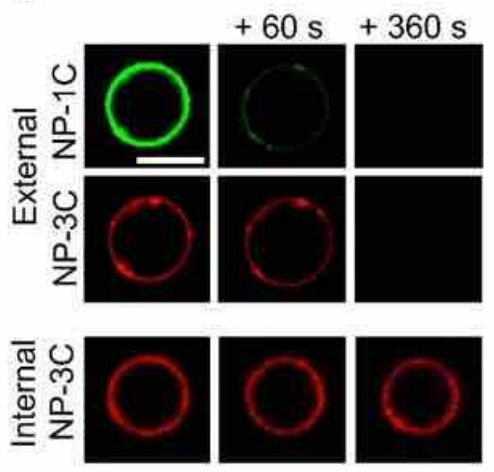

B

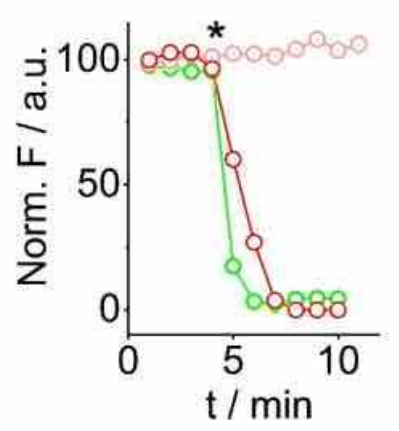

C

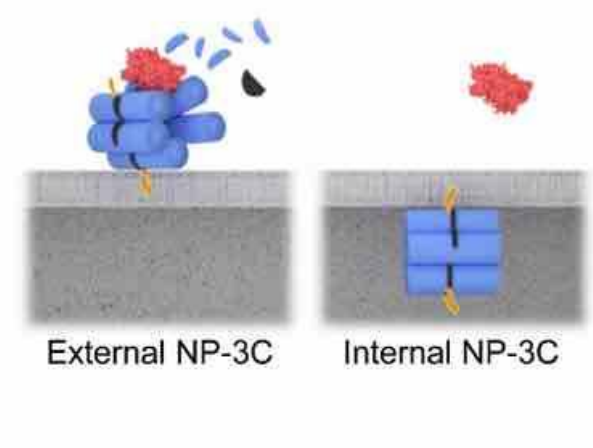

Figure 6. $N P-3 C$ predominately binds in a membrane-adhering orientation to low-curvature membranes. (A) Confocal laser scanning microscopic images of a GUV with externally bound NP-1C (FAM, top row) and NP-3C (Cy3, bottom row) before and after addition of BAL-31 (1.7 $U$ ) and incubation for the indicated durations, and internally located NP-3C (Cy3) before and after the addition of BAL-31 (8.2 U). Scale bar, $10 \mu \mathrm{m}$. (B) Kinetic profiles for the digestion of $N P-1 C$ (green) and NP-3C (red) (data from (A). The arrow indicates the addition of the nuclease probe. (C) Schematic drawing of NP-3C tethered to the low-curvature membrane of a GUV. The orientation of the DNA pore leaves the nicks accessible to the nuclease thereby increasing digestion for the external nanopore, but is inaccessible to the internal nanopore.

Expanding the digestion assay to other membrane-bound DNA nanostructures. To showcase the wider applicability of the nuclease probe assay, we engineered a DNA origami plate containing cholesterol anchors capable to tethering the plates to membranes. We utilized a previously published $50 \times 50 \mathrm{~nm}$ plate $^{46}$ and equipped it with $0,1,2$ and 4 cholesterols to identify any membrane interaction with SUVs (Figure S14). All cholesterol-modified plates 
interacted tightly with SUVs as demonstrated by gel electrophoresis (Figure S15). The binding took place despite the about 100-times smaller size of the cholesterol tag compared to the DNA plate. To study the nanostructures' susceptibility to the nuclease probe, we acquired a kinetic digestion profiles for plates with 4 and no cholesterols in the absence and presence of SUVs (Figure S16). The read-out revealed that cholesterol-modified plates received protection from membrane binding compared to the non-modified version. The protection stems from the steric blockade by the membrane to block the digestion of the DNA nanostructure from its membranebound side.

\section{CONCLUSIONS}

The ability of DNA nanopores to modulate membranes is of fundamental scientific interest and considerable impact in biosensing, synthetic biology, and cell biological research. $22,24,25,27,31,47,48$ Yet, there is a lack of knowledge on mechanistic steps leading to membrane insertion, the extent of membrane puncturing, and the influence of membrane curvature and lipid anchors. In addition, DNA nanopores can suffer from low insertion rates into routinely used planar lipid bilayers. Up until now, most reports have not been able to address these questions as they have primarily focused on the construction of the pores and their transport properties.

In this study, we set out to gain a deeper understanding of how an archetypal DNA nanopore with up to three cholesterol anchors interacts with lipid bilayers of various membrane curvatures. Our results can be summarized in the following highlights: (i) A nuclease probe assay was developed to identify the orientation of the DNA nanopore relative to the membrane. Based on the differential accessibility of a nuclease probe, the assay distinguishes between pores in a membrane-spanning or membrane-tethered orientation that have either low or high nuclease 
digestion rates, respectively. How does the strategy compare to other approaches? While fluorescence resonance energy transfer (FRET) analysis has not been applied for DNA porebilayer interactions so far, the nuclease probe assay certainly has a comparably high structural resolution and is potentially also universal to be implemented with many different membrane systems including cells and also semifluid polymeric membranes. ${ }^{47}$

As a further finding, we discovered that (ii) membrane tethering requires one cholesterol anchor, while membrane insertion proceeds with three tags. How does this compare to previous studies? So far, membrane-spanning has been achieved with two hydrophobic phophyrins, ${ }^{26} 72$ ethylated phosphorothiates, ${ }^{23}$ and $2-4$ cholesterol anchors, ${ }^{29,38}$ but also numerous cholesterol ${ }^{22}$ and tocophero ${ }^{30}$ tags that integrate into the bilayer with its polar head group region. The advantage of our three-cholesterol pore design is its structural simplicity, symmetry, and rapid ease of assembly. This will facilitate future computational studies ${ }^{49,50}$ to answer the puzzling question on how lipids arrange around the biophysically unusual outer pore wall with an alternating pattern of hydrophobic cholesterol tags and hydrophilic phosphate backbone groups.

In finding (iii), the nanopore inserts into membranes via a two-step mechanism (Figure 7). The first step involves membrane tethering, and the second step for membrane spanning. Under our experimental conditions, binding proceeds rapidly $(<5 \mathrm{~min})$, while the transition to the spanning pore on the ensemble occurs at a time scale of around $1 \mathrm{~h}$. By comparison, previous reports have characterized the result of insertion, the membrane-spanning pore. The kinetics for insertion is surprisingly slow but in line with the overall low insertion frequency reported for DNA nanopores when analyzed with planar lipid bilayer recordings. ${ }^{23,30}$ 


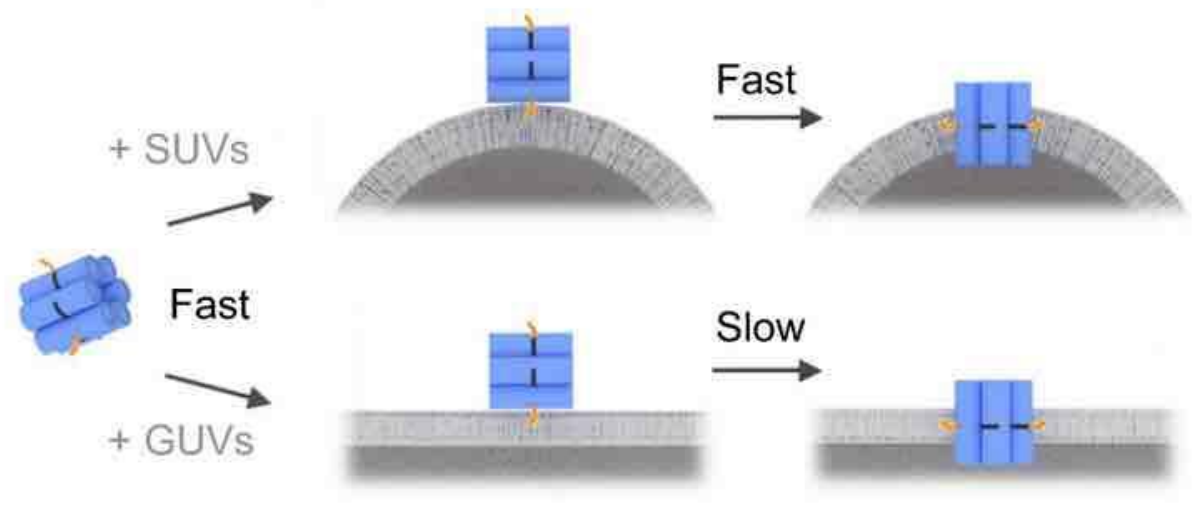

Figure 7. Inferred mechanism for membrane tethering and insertion of DNA nanopore NP-3C with three cholesterol anchors. Initial membrane tethering is fast for both SUVs and GUVs, but full bilayer insertion only occurs readily for membranes with a high curvature, such as SUVs, yet significantly slower for low-curvature GUVs or planar membranes.

As other observations, (iv) membrane insertion is an efficient process for the used bilayers with high curvature $\left(20 \mu \mathrm{m}^{-1}\right)$, while (v) lipid vesicles with a 100-fold lower curvature $\left(0.2 \mu \mathrm{m}^{-1}\right)$ resulted in poor insertion, leaving the DNA nanostructure in a membrane-tethered orientation. The preferential insertion of DNA nanopores into high-curvature membranes is most likely due to the highly strained lipid arrangement in strongly curved membranes. ${ }^{40}$ Probable defect regions within the lipid packing may accommodate partial DNA pore insertion, followed by subsequent dynamic lipid rearrangement towards completely spanning pores in high ratios. By contrast, insertion into a structurally more regular planer bilayer would likely incur a much higher energetic penalty due to the greater deformation required for opening up voids in flatter lipid bilayers. We expect that this effect will depend on the lipid composition of the membrane.

Our study will impact the field of membrane-interacting DNA nanostructures in several ways. Of practical relevance, the previously reported problem of poor insertion of DNA 
nanopores can be tackled by using highly curved membranes or planar membrane that contain due to their lipid composition- structural mismatches that facilitate insertion. Alternatively, DNA nanopores might be designed that deliberately deform lipid bilayers to facilitate insertion. In addition, our nuclease probe approach can become a more generic tool to investigate the topography of many other DNA nanostructures with membranes of varying lipid compositions, and even potentially for investigating interactions with cells. In conclusion, the nuclease assay and the fundamental findings will likely help advance this emerging field of membraneinteracting DNA nanostructures. 


\section{METHODS/EXPERIMENTAL}

Design and Folding DNA Nanostructures. The DNA nanopore was published previously, information on the DNA oligonucleotide sequences, two-dimensional DNA maps and pore dimensions is provided in the supporting information (Tables S1 and S2, Figure S1). To fold the nanostructures, an equimolar mixture of DNA oligonucleotides (Integrated DNA Technologies, US) (1 nmol of each strand) was dissolved in phosphate buffered saline (Sigma, UK) $(1 \times$ PBS, total volume $250 \mu \mathrm{L})$ at room temperature, and then incubated at $95{ }^{\circ} \mathrm{C}$ for $2 \mathrm{~min}$, followed by cooling to $20^{\circ} \mathrm{C}$ at a rate of $5{ }^{\circ} \mathrm{C}$ per min.

Vesicle Preparation. DPhPC small unilamellar vesicles (SUVs): a solution of 1,2diphytanoyl-sn-glycero-3-phosphocholine (Avanti Polar Lipid Inc., US) (DPhPC, 10 mM) containing cholesterol (Sigma, UK) $(1 \mathrm{mM})$ in chloroform $(200 \mu \mathrm{L})$ was added to an oven-dried round bottom flask $(10 \mathrm{~mL})$. The solvent was removed under rotation and vacuum using a rotary evaporator for $20 \mathrm{~min}$, after which the thin film was dried under ultrahigh vacuum for $3 \mathrm{~h}$. $1 \mathrm{x}$ phosphate buffered saline (PBS) $(1 \mathrm{~mL})$ was added and the solution was sonicated using a bath sonicator for $20 \mathrm{~min}$. SUVs were stored in the fridge and used within 1 week. Before experimentation, the SUV solution was vortexed for $2 \mathrm{~s}$. SUVs were subjected to dynamic light scattering to confirm the diameter of the vesicles using a Zetasizer Nano (Malvern, UK). DPhPC giant unilamellar vesicles (GUVs) were prepared by following a modified published protocol. ${ }^{30}$ DPhPC $(10 \mathrm{mM})$ containing cholesterol $(1 \mathrm{mM})$ in chloroform $(150 \mu \mathrm{L})$ was added to a small glass vial $(1 \mathrm{~mL})$, and the solvent was removed under rotation and vacuum using a rotary evaporator for $20 \mathrm{~min}$. The thin film was re-suspended in mineral oil (Sigma, UK) $(150 \mu \mathrm{L}$ ), followed by votexing for $2 \mathrm{~min}$. The solution was shaken on a thermomixer (Eppendorf, UK) $800 \mathrm{RPM}$ for $1 \mathrm{~h}$ at room temperature, then transferred to a $1 \mathrm{~mL}$ plastic vial. Sucrose $(400 \mathrm{mM}$, 
$25 \mu \mathrm{L})$ was then added and the solution vortexed for $2 \mathrm{~min}$, after which glucose (400 $\mathrm{mM}, 150$ $\mu \mathrm{L})$ was carefully added to the top of the solution to generate two phases. The vial was then centrifuged at 6'000 RPM for $20 \mathrm{~min}$ at room temperature, the supernatant carefully removed. An additional glucose $(400 \mathrm{mM}, 150 \mu \mathrm{L})$ washing step was performed, the vial centrifuged for $10 \mathrm{~min}$ and the supernatant removed. Finally, glucose $(400 \mathrm{mM}, 150 \mu \mathrm{L})$ was added and the vial gently mixed to re-suspend the GUVs. For confocal studies, the GUVs were used within $4 \mathrm{~h}$ of preparation. For the internal encapsulation of Cy3-labelled NP-3C with GUVs, NP-3C (2.5 $\mu \mathrm{L}, 1$ $\mu \mathrm{M})$ was added to Sucrose $(400 \mathrm{mM}, 25 \mu \mathrm{L})$ containing $\mathrm{KCl}(500 \mathrm{mM})$.

Agarose Gel Electrophoresis. The SUV binding assays were analyzed using a $0.5 \%$ agarose gel in TAE buffer $\mathrm{pH}$ 8.0. The low percentage agarose was required to move NP-1C onto the gel phase. A solution containing 2 pmol NP-0C or NP-1C, was mixed with PBS / SUV (25 $\mu \mathrm{L}$ total volume), the solution was gently mixed at room temperature for $1 \mathrm{~h}$. Purple gel loading dye (5 $\mu \mathrm{L}$, New England Biolabs, UK) was added to the solution, of which $20 \mu \mathrm{L}$ was transferred into the wells of the gel. The gel was run at $30 \mathrm{~V}$ for $45 \mathrm{~min}$ at $8{ }^{\circ} \mathrm{C}$. The bands were visualized by ultraviolet illumination after staining with ethidium bromide solution. A 100 or 1000-base-pair marker (New England Biolabs, UK) was used as the reference standard. The digestion of NP-0C using BAL-31 was performed using a $2 \%$ agarose gel to resolve the fragmentation. To NP-0C (60 $\mu \mathrm{L}, 1 \mu \mathrm{M}$, in PBS), $1 \mathrm{X}$ BAL buffer (New England Biolabs, UK)

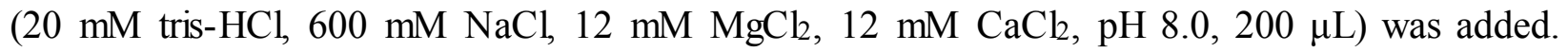
After 5 min, BAL-31 (New England Biolabs, UK) (9.6 U), was added and the solution gently mixed at room temperature. $20 \mu \mathrm{L}$ fractions were taken at the stated time points, and quickly transferred into a small plastic vial containing purple gel loading dye (New England Biolabs, UK) $(10 \mu \mathrm{L})$, pre-cooled on ice. $20 \mu \mathrm{L}$ of this solution was then transferred to the gel, and the gel 
was run at $60 \mathrm{~V}$ for $50 \mathrm{~min}$ at $8{ }^{\circ} \mathrm{C}$. The bands were visualized by ultraviolet illumination after staining with ethidium bromide solution. A 100 or 1000-base-pair marker (New England Biolabs, UK) was used as the reference standard.

UV Absorption Spectroscopy and Melting Temperature Analysis. UV absorption digestion profiles and melting temperatures $\mathrm{T}_{\mathrm{m}}$ of the nanostructures was determined using a Varian Cary 300 Bio UV-vis spectrophotometer equipped with a Peltier element and a quartz cuvette with a path length of $1 \mathrm{~cm}$. The peak absorbance band at $260 \mathrm{~nm}$ was monitored using a scan rate of $600 \mathrm{~nm}$ per minute. The DNA nanostructures (70 $\mu \mathrm{L}, 1 \mu \mathrm{M}$, PBS) were added to $1 \mathrm{X}$ BAL buffer $(630 \mu \mathrm{L})$ and the experiment performed after 2 min of thermal equilibration. As stated, BAL-31 (13 U) was added and the solution quickly stirred. Melting studies were performed heating at a rate of $2{ }^{\circ} \mathrm{C}$ per min from $20-80{ }^{\circ} \mathrm{C}$, collecting data every $1{ }^{\circ} \mathrm{C}$.

Fluorescence Spectroscopy. The Cy3 emission peaks of the nanostructures were monitored using a Varian Eclipse fluorescence spectrophotometer. The samples were analyzed by excitation at $540 \mathrm{~nm}$, and the emission monitored kinetically at $563 \mathrm{~nm}$, or scan range between 550 to $750 \mathrm{~nm}$. A 5-10 mm slit width and 600-800 PMT voltage was used, scanning at a rate of $600 \mathrm{~nm}$ per min, using quartz cuvette with a path length of $1 \mathrm{~cm}$. The DNA nanostructures $(2 \mu \mathrm{L}, 1 \mu \mathrm{M}$, in PBS) were added to PBS / vesicles (18 $\mu \mathrm{L}, 1 \mathrm{mM}$, in PBS) and incubated for $1 \mathrm{~h}$ (unless stated otherwise) at room temperature in a $1 \mathrm{~mL}$ plastic vial. BAL buffer $(130 \mu \mathrm{L})$ was then added to the solution, and then promptly transferred to the cuvette. After $2 \mathrm{~min}$ of thermal equilibration, BAL $(0.25 \mu \mathrm{L}$, stock $0.25 \mathrm{U}$ per $\mathrm{mL}$, unless stated otherwise) was added and the solution rapidly stirred. For the data in Figure 2, the constructs were measured in 3 separate cuvettes, either with or without SUVs in parallel. For all other experiments, the data was collected in the same cuvette. 
Confocal Laser Scanning Microscopy. Images were collected using a confocal laser scanning microscope (60x objective, FV-1000, Olympus, UK). Images were analyzed using ImageJ software. The DNA constructs were combined $(0.5 \mu \mathrm{L}$ each, stock $1 \mu \mathrm{M})$, and added to GUVs $(5 \mu \mathrm{L}, \sim 2 \mathrm{mg}$ per $\mathrm{mL}, 400 \mathrm{mM}$ glucose), and gently mixed at room temperature for $1 \mathrm{~h}$. Prior to experimentation, the tissue culture dish with cover glass bottom (23 mm diameter, 0.17 mm thickness, World Precision Instruments, UK) was pre-washed with bovine serum albumin (Sigma, UK) $(10 \mathrm{mg}$ per $\mathrm{mL}, 100 \mu \mathrm{L})$, followed by PBS $(3 \times 100 \mu \mathrm{L})$. The DNA-GUV solution was then added to $1 \mathrm{x}$ BAL buffer $(50 \mu \mathrm{L})$, and the vesicles allowed to settle onto the microscope slide for $10-20 \mathrm{~min}$. BAL-31 (0.4 $\mu \mathrm{L}$, unless stated otherwise) was carefully added to the top of the droplet, as close to the region of interest as possible without disturbing the vesicles. For the internal NP-3C GUV assay, the external BAL-31 buffer was supplemented with additional $\mathrm{KCl}$ $(500 \mathrm{mM})$ in order to get the GUVs to settle on the confocal slide (note, BAL-31 remained active at this higher salt concentration).

Single channel current recordings. The recordings were performed following a modified version of a published protocol ${ }^{51}$. Using a chip-based, parallel bilayer recording set-up (Orbit 16, Nanion Technologies, Munich, Germany) with multi-electrode-cavity-array (MECA) chips (Ionera Technologies, DE), and electrolyte solution (150 $\mu \mathrm{L}, 1 \mathrm{M} \mathrm{KCl}, 10 \mathrm{mM}$ HEPES pH 8.0, $150 \mu \mathrm{L} 1 \mathrm{x}$ BAL buffer). Bilayers were automatically formed by remotely actuated spreading of DPhPC dissolved in hexane $\left(1 \mathrm{mg} \mathrm{m}^{-1}\right)$. For pore insertion, a 2:1 mixture of NP-3C and $0.5 \%$ n-octyl-oligo-oxyethylene dissolved in PBS was added to the cis side of the bilayer to a final concentration of 10-50 $\mathrm{nM}$ nanopores. A positive voltage of +50 or $+100 \mathrm{mV}$ was applied to facilitate pore insertion. Once a stable nanopore channel was observed, BAL-31 (3.3 U) was 
added to the cis chamber and the conductance monitored. The ionic current data were Bessel filtered at $2.07 \mathrm{kHz}$ and acquired at $20 \mathrm{kHz}$ using an EPC-10 patch-clamp amplifier (HEKA Elektronik, DE) with the PATCHMASTER software (HEKA Elektronik, DE). Single channel analysis was performed using Clampfit (Molecular Devices, CA, USA). 
Supporting Information. DNA sequences, combinations of oligonucleotides used to generate each construct, 2D maps and 3D renderings, as well as additional experimental data on digestion, and control CLSM studies is available free of charge via the Internet.

\section{AUTHOR INFORMATION}

Corresponding Authors

jonathan.burns@ucl.ac.uk, s.howorka@ucl.ac.uk

Author Contributions

J.B. designed and carried out all experiments, and J.B. and S.H wrote the manuscript.

Funding Sources

S.H. is supported by the EPSRC (EP/N009282/1), the BBSRC (BB/M012700/1, BB/M025373/1, BB/N017331/1), and the Leverhulme Trust (RPG-2017-015).

\section{REFERENCES}

1. Dal Peraro, M.; van der Goot, F. G., Pore-Forming Toxins: Ancient, but Never Really out of Fashion. Nat. Rev. Microbiol. 2016, 14, 77-92.

2. Pagès, J. M.; James, C. E.; Winterhalter, M., The Porin and the Permeating Antibiotic: A Selective Diffusion Barrier in Gram-Negative Bacteria. Nat.Rev. Microbiol. 2008, 6, 893-903.

3. Yeager, M.; Harris, A. L., Gap Junction Channel Structure in the Early $21^{\text {st }}$ Century: Facts and Fantasies. Curr. Opin. Cell Biol. 2007, 19, 521-528. 
4. Kosinski, J.; Mosalaganti, S.; von Appen, A.; Teimer, R.; DiGuilio, A. L.; Wan, W.; Bui, K. H.; Hagen, W. J.; Briggs, J. A.; Glavy, J. S.; Hurt, E.; Beck, M., Molecular Architecture of the Inner Ring Scaffold of the Human Nuclear Pore Complex. Science 2016, 352, 363-365.

5. Lin, D. H.; Stuwe, T.; Schilbach, S.; Rundlet, E. J.; Perriches, T.; Mobbs, G.; Fan, Y.; Thierbach, K.; Huber, F. M.; Collins, L. N.; Davenport, A. M.; Jeon, Y. E.; Hoelz, A., Architecture of the Symmetric Core of the Nuclear Pore. Science 2016, 352, aafl 015.

6. Banghart, M.; Borges, K.; Isacoff, E.; Trauner, D.; Kramer, R. H., Light-Activated Ion Channels for Remote Control of Neuronal Firing. Nat. Neurosci. 2004, 7, 1381-1386.

7. Volgraf, M.; Gorostiza, P.; Numano, R.; Kramer, R. H.; Isacoff, E. Y.; Trauner, D., Allosteric Control of an Ionotropic Glutamate Receptor with an Optical Switch. Nat. Chem. Biol. 2006, 2, 47-52.

8. Quick, J.; Loman, N. J.; Duraffour, S.; Simpson, J. T.; Severi, E.; Cowley, L.; Bore, J. A.; Koundouno, R.; Dudas, G.; Mikhail, A.; Ouedraogo, N.; Afrough, B.; Bah, A.; Baum, J. H.; Becker-Ziaja, B.; Boettcher, J. P.; Cabeza-Cabrerizo, M.; Camino-Sanchez, A.; Carter, L. L.; Doerrbecker, J., et al., Real-Time, Portable Genome Sequencing for Ebola Surveillance. Nature 2016, 530, 228-232.

9. Larkin, J.; Henley, R. Y.; Jadhav, V.; Korlach, J.; Wanunu, M., Length-Independent DNA Packing into Nanopore Zero-Mode Waveguides for Low-Input DNA Sequencing. Nat. Nanotechnol. 2017.

10. Howorka, S.; Siwy, Z., Nanopore Analytics: Sensing of Single Molecules. Chem. Soc. Rev. 2009, 38, 2360-2384.

11. Wang, G.; Wang, L.; Han, Y.; Zhou, S.; Guan, X., Nanopore Stochastic Detection: Diversity, Sensitivity, and Beyond. Acc. Chem. Res. 2013, 46, 2867-2877. 
12. Stoloff, D. H.; Wanunu, M., Recent Trends in Nanopores for Biotechnology. Curr. Opin. Biotechnol. 2013, 24, 699-704.

13. Reiner, J. E.; Balijepalli, A.; Robertson, J. W. F.; Campbell, J.; Suehle, J.; Kasianowicz, J. J., Disease Detection and Management Via Single Nanopore-Based Sensors. Chem. Rev. 2012, $112,6431-6451$.

14. Miles, B. N.; Ivanov, A. P.; Wilson, K. A.; Dogan, F.; Japrung, D.; Edel, J. B., Single Molecule Sensing with Solid-State Nanopores: Novel Materials, Methods, and Applications. Chem. Soc. Rev. 2013, 42, 15-28.

15. Venkatesan, B. M.; Bashir, R., Nanopore Sensors for Nucleic Acid Analysis. Nat. Nanotechnol. 2011, 6, 615-624.

16. Wei, R. S.; Gatterdam, V.; Wieneke, R.; Tampe, R.; Rant, U., Stochastic Sensing of Proteins with Receptor-Modified Solid-State Nanopores. Nat. Nanotechnol. 2012, 7, 257-263.

17. Heerema, S. J.; Dekker, C., Graphene Nanodevices for DNA Sequencing. Nat. Nanotechnol. 2016, 11, 127-136.

18. Feng, J.; Liu, K.; Bulushev, R. D.; Khlybov, S.; Dumcenco, D.; Kis, A.; Radenovic, A., Identification of Single Nucleotides in Mos2 Nanopores. Nat. Nanotechnol. 2015, 10, 10701076.

19. Wang, Y.; Zheng, D. L.; Tan, Q. L.; Wang, M. X.; Gu, L. Q., Nanopore-Based Detection of Circulating Micrornas in Lung Cancer Patients. Nat. Nanotechnol. 2011, 6, 668-674.

20. Yusko, E. C.; Bruhn, B. R.; Eggenberger, O. M.; Houghtaling, J.; Rollings, R. C.; Walsh, N. C.; Nandivada, S.; Pindrus, M.; Hall, A. R.; Sept, D.; Li, J.; Kalonia, D. S.; Mayer, M., RealTime Shape Approximation and Fingerprinting of Single Proteins Using a Nanopore. Nat. Nanotechnol. 2017, 12, 360-367. 
21. Bell, N. A.; Engst, C. R.; Ablay, M.; Divitini, G.; Ducati, C.; Liedl, T.; Keyser, U. F., DNA Origami Nanopores. Nano Lett. 2012, 12, 512-517.

22. Langecker, M.; Arnaut, V.; Martin, T. G.; List, J.; Renner, S.; Mayer, M.; Dietz, H.;

Simmel, F. C., Synthetic Lipid Membrane Channels Formed by Designed DNA Nanostructures.

Science 2012, 338, 932-936.

23. Burns, J.; Stulz, E.; Howorka, S., Self-Assembled DNA Nanopores That Span Lipid Bilayers. Nano Lett. 2013, 13, 2351-2356.

24. Howorka, S., Nanotechnology. Changing of the Guard. Science 2016, 352, 890-891.

25. Howorka, S., Building Membrane Nanopores. Nat. Nanotechnol. 2017, 12, 619-630.

26. Burns, J. R.; Göpfrich, K.; Wood, J. W.; Thacker, V. V.; Stulz, E.; Keyser, U. F.;

Howorka, S., Lipid Bilayer-Spanning DNA Nanopores with a Bifunctional Porphyrin Anchor. Angew. Chem. Int. Ed. 2013, 52, 12069-12072.

27. Burns, J. R.; Al-Juffali, N.; Janes, S. M.; Howorka, S., Membrane-Spanning DNA Nanopores with Cytotoxic Effect. Angew. Chem. Int. Ed. 2014, 53, 12466-12470.

28. Seifert, A.; Göpfrich, K.; Burns, J. R.; Fertig, N.; Keyser, U. F.; Howorka, S., BilayerSpanning DNA Nanopores with Voltage-Switching between Open and Closed State. ACS Nano 2015, 9, 1117-1126.

29. Gopfrich, K.; Zettl, T.; Meijering, A. E.; Hernandez-Ainsa, S.; Kocabey, S.; Liedl, T.; Keyser, U. F., DNA-Tile Structures Induce Ionic Currents through Lipid Membranes. Nano Lett. 2015, 15, 3134-3138.

30. Krishnan, S.; Ziegler, D.; Arnaut, V.; Martin, T. G.; Kapsner, K.; Henneberg, K.; Bausch, A. R.; Dietz, H.; Simmel, F. C., Molecular Transport through Large-Diameter DNA Nanopores. Nat. Commun. 2016, 7, 12787. 
31. Gopfrich, K.; Li, C. Y.; Ricci, M.; Bhamidimarri, S. P.; Yoo, J.; Gyenes, B.; Ohmann, A.; Winterhalter, M.; Aksimentiev, A.; Keyser, U. F., Large-Conductance Transmembrane Porin Made from DNA Origami. ACS Nano 2016, 10, 8207-8214.

32. Seeman, N. C., Nanomaterials Based on DNA. Annu. Rev. Biochem. 2010, 79, 65-87.

33. Rothemund, P. W., Folding DNA to Create Nanoscale Shapes and Patterns. Nature 2006, 440, 297-302.

34. Chen, Y. J.; Groves, B.; Muscat, R. A.; Seelig, G., DNA Nanotechnology from the Test Tube to the Cell. Nat. Nanotechnol. 2015, 10, 748-760.

35. Pinheiro, A. V.; Han, D.; Shih, W. M.; Yan, H., Challenges and Opportunities for Structural DNA Nanotechnology. Nat. Nanotechnol. 2011, 6, 763-772.

36. Jones, M. R.; Seeman, N. C.; Mirkin, C. A., Nanomaterials. Programmable Materials and the Nature of the DNA Bond. Science 2015, 347, 1260901.

37. Douglas, S. M.; Marblestone, A. H.; Teerapittayanon, S.; Vazquez, A.; Church, G. M.; Shih, W. M., Rapid Prototyping of 3d DNA-Origami Shapes with caDNAno. Nucleic Acids Res. 2009, 37, 5001-5006.

38. Burns, J. R.; Seifert, A.; Fertig, N.; Howorka, S., A Biomimetic DNA-Based Channel for the Ligand-Controlled Transport of Charged Molecular Cargo across a Biological Membrane. Nat. Nanotechnol. 2016, 11, 152-156.

39. Walker, B.; Bayley, H., Key Residues for Membrane Binding, Oligomerization, and Pore Forming Activity of Staphylococcal Alpha-Hemolysin Identified by Cysteine Scanning Mutagenesis and Targeted Chemical Modification. J. Biol. Chem. 1995, 270, 23065-23071.

40. Jarsch, I. K.; Daste, F.; Gallop, J. L., Membrane Curvature in Cell Biology: An Integration of Molecular Mechanisms. J. Cell Biol. 2016, 214, 375-387. 
41. McMahon, H. T.; Boucrot, E., Membrane Curvature at a Glance. J. Cell Sci. 2015, 128, 1065-1070.

42. Peter, B. J.; Kent, H. M.; Mills, I. G.; Vallis, Y.; Butler, P. J.; Evans, P. R.; McMahon, H. T., BAR Domains as Sensors of Membrane Curvature: The Amphiphysin BAR Structure.

Science 2004, 303, 495-499.

43. Maingi, V.; Lelimousin, M.; Howorka, S.; Sansom, M. S., Gating-Like Motions and Wall Porosity in a DNA Nanopore Scaffold Revealed by Molecular Simulations. ACS Nano 2015, 9, 11209-11217.

44. Gray, H. B., Jr.; Ostrander, D. A.; Hodnett, J. L.; Legerski, R. J.; Robberson, D. L., Extracellular Nucleases of Pseudomonas Bal 31. I. Characterization of Single Strand-Specific Deoxyriboendonuclease and Double-Strand Deoxyriboexonuclease Activities. Nucleic Acids Res. 1975, 2, 1459-1492.

45. Legerski, R. J.; Hodnett, J. L.; Gray, H. B., Jr., Extracellular Nucleases of Pseudomonas Bal 31. Iii. Use of the Double-Strand Deoxyriboexonuclease Activity as the Basis of a Convenient Method for the Mapping of Fragments of DNA Produced by Cleavage with Restriction Enzymes. Nucleic Acids Res. 1978, 5, 1445-1464.

46. Hager, R.; Halilovic, A.; Burns, J. R.; Schaffler, F.; Howorka, S., Arrays of Individual DNA Molecules on Nanopatterned Substrates. Sci. Rep. 2017, 7.

47. Messager, L.; Kim, J.; Burns, J. R.; Cecchin, D.; Hindley, J.; Pyne, A.; Gaitzsch, J.; Battaglia, G.; Howorka, S., Biomimetic Hybrid Nanocontainers of Designed Permeability. Angew. Chem. Int. Ed. 2016, 55, 11106-11109. 
48. Gopfrich, K.; Li, C. Y.; Mames, I.; Bhamidimarri, S. P.; Ricci, M.; Yoo, J.; Mames, A.; Ohmann, A.; Winterhalter, M.; Stulz, E.; Aksimentiev, A.; Keyser, U. F., Ion Channels Made from a Single Membrane-Spanning DNA Duplex. Nano Lett. 2016, 16, 4665-4669.

49. Maingi, V.; Burns, J. R.; Uusitalo, J. J.; Howorka, S.; Marrink, S. J.; Sansom, M. S., Stability and Dynamics of Membrane-Spanning DNA Nanopores. Nat. Commun. 2017, 8, 14784.

50. Maffeo, C.; Bhattacharya, S.; Yoo, J.; Wells, D.; Aksimentiev, A., Modeling and Simulation of Ion Channels. Chem. Rev. 2012, 112, 6250-6284.

51. Del Rio Martinez, J. M.; Zaitseva, E.; Petersen, S.; Baaken, G.; Behrends, J. C., Automated Formation of Lipid Membrane Microarrays for Ionic Single-Molecule Sensing with Protein Nanopores. Small 2015, 11, 119-125. 


\section{TOC graphic}

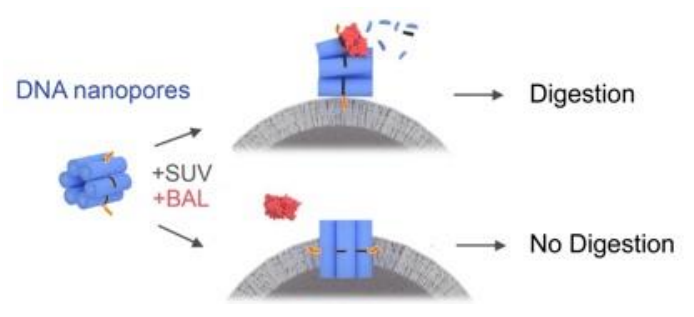

The orientation of DNA nanopores relative to a lipid bilayer is determined with a nuclease probe assay that digests adhering but not membrane-puncturing pores. 